\title{
Manifestaciones clínicas durante exposición a altas fuerzas $G$ en centrifuga humana
}

\author{
Alonso Rodríguez C. ${ }^{1}$, Medina Font J. ${ }^{2}$, Puente Espada B. ${ }^{3}$
}

Sanid. mil. 2012; 68 (3): 157-162; ISSN: 1887-8571

\begin{abstract}
RESUMEN
Introducción: La exposición del hombre a altos niveles de aceleraciones origina la aparición de síntomas y signos clínicos, incluyendo el riesgo de pérdida de conciencia que puede comprometer la seguridad en vuelo, constituyendo el entrenamiento en centrifuga humana un método útil para mejorar la tolerancia a las altas aceleraciones. Material y métodos: En un grupo de 81 pilotos de combate que recibieron entrenamiento en una centrifuga humana avanzada, exponiéndose a un nivel de aceleración de +7 Gz durante 15 segundos, con una velocidad de comienzo de $6 \mathrm{G} /$ segundo, se evaluó la aparición de síntomas y signos. También se estudió la tolerancia individual relajada a las aceleraciones. Resultados: Del total de la población de pilotos, 96,3\% experimentó síntomas visuales (visión gris 88,9\%, visión en túnel $72,9 \%$ y visión negra $39,5 \%$ ). El 90,1\% presentó petequias cutáneas, $88,6 \%$ astenia y $75,8 \%$ inestabilidad y vértigo. Un $60,5 \%$ refirió haber tenido dolores musculares en antebrazos, $58 \%$ en muslos, $40,7 \%$ en brazos, $30,9 \%$ en cuello, $23,5 \%$ en abdomen, $20,5 \%$ en tórax y $20,3 \%$ en codos. También se determinó en ellos la tolerancia relajada al aumento gradual de aceleraciones en la centrífuga, estableciéndose en $4,79 \pm 0,81+\mathrm{Gz}$, oscilando entre 3,40+Gz el de menor tolerancia y 6,86+Gz el de mayor. Conclusiones: Comparando el nivel de tolerancia individual relajada con los síntomas hallados, se observa como los pilotos con mayor y menor tolerancia natural a las fuerzas Gz experimentaron visión negra con mayor frecuencia que aquéllos con tolerancia intermedia. No se han encontrado diferencias significativas entre el nivel de tolerancia individual a las fuerzas Gz y la frecuencia de aparición de petequias, astenia, mareos y dolores músculo-esqueléticos de diversa localización.
\end{abstract}

PALABRAS CLAVE: Aceleraciones, Visión gris, Visión negra, Petequias, Fatiga, Desorientación, Pérdida de conocimiento, Centrifuga humana.

\section{Clinical findings during high $\mathrm{g}$ forces exposure in human centrifuge SUMMARY:}

Introduction: The man exposure to high acceleration level produces the appearance of signs and symptoms, including the risk of loss of consciousness, that may compromise flight safety, and the training in human centrifuge is an useful method to improve the high acceleration tolerance. Material and methods: We assessed the appearance of symptoms and signs in a group of 81 fighter pilots who underwent advanced human centrifuge training, by exposing themselves to a $7 \mathrm{Gz}$ for 15 seconds, with an on-set rate of $6 \mathrm{G} /$ second. We also studied the individual relaxed G tolerance level. Results: Visual symptoms were reported by $96,3 \%$ (greyout $88,9 \%$, tunnel vision $72,9 \%$, blackout $39,5 \%$ ). We found that $90,1 \%$ presented petechials hemorrhages, $8,6 \%$ reported fatigue and 75 ' $8 \%$ disorientation or vertigo. Musculoskeletal pain was commonly reported affecting to different body parts: to forearms in $60,5 \%$, to the thighs in $58 \%$, to the arms in $40,7 \%$, to the neck in $30,9 \%$, to the abdomen in $23,5 \%$, to the chest in $20,5 \%$ and to the elbows in $20,3 \%$ of the pilots. The average relaxed G-level tolerance was established in $4,79 \pm 0,81 \mathrm{Gz}$, with a range between $3,40 \mathrm{Gz}$ and $6,86 \mathrm{Gz}$, by using a centrifuge gradual on-set rate. Conclusions: Comparing the $\mathrm{G}$ level tolerance with the symptoms and signs, we found a significant greater prevalence of blackout among pilots with the biggest and the least tolerance than among those pilots with intermediate tolerance. We have not found any differences between the G level tolerance and the prevalence of petechiae, weariness, vertigo or musculoskeletal aches of different locations.

KEY WORDS: Accelerations, Grey out, Blackout, Petechiae, Fatigue, Disorientation, Loss of consciousness, Human centrifuge.

\section{INTRODUCCIÓN}

El vuelo en aviones de combate es la única actividad humana que expone de forma prolongada al hombre a niveles de aceleración varias veces superiores a la de la gravedad terrestre. La aceleración se

\section{${ }^{1}$ GB. Médico.}

${ }^{2}$ Doctor en Farmacia.

${ }^{3}$ Cte. Médico.

Centro de Instrucción de Medicina Aeroespacial (CIMA).

Dirección para correspondencia: caloro1@oc.mde.es

Recibido: 12 de diciembre de 2011

Aceptado: 9 de marzo de 2012 define como cambio de velocidad de un objeto en función del tiempo y como la velocidad es una magnitud vectorial dotada de intensidad dirección, sentido y punto de aplicación, la aceleración resulta, bien de un cambio de velocidad en una línea recta (aceleración lineal), bien del cambio en la dirección de desplazamiento (aceleración radial). Las aceleraciones se cuantifican en unidades $G$, unidad que equivale a la aceleración de la gravedad terrestre, que es constante y de valor $9,81 \mathrm{~m} / \mathrm{seg}^{2}$. Las aceleraciones, en función de la tercera ley de Newton, provocan una reacción de la misma intensidad, dirección y sentido opuesto, generando una fuerza de inercia que actúa sobre el piloto en distintas direcciones, en función de las cuáles producen diferentes efectos. En este artículo se referirá a la componente que 
actúa a lo largo del eje longitudinal del cuerpo humano o eje $z$, en sentido cráneo-caudal y que se denominan aceleraciones $+\mathrm{Gz}^{1-3}$.

La exposición a fuerzas $\mathrm{Gz}$ desencadena una serie de respuestas fisiológicas destinadas a compensar la pérdida de perfusión cerebral y prevenir la hipoxia cerebral ${ }^{4}$, pero éstos mecanismos por sí solos son insuficientes por encima de un determinado nivel de aceleraciones para prevenir la pérdida de conocimiento, siendo necesario dotar a los pilotos de un entrenamiento físico ${ }^{5}$, de un equipo personal específico ${ }^{6,7}$ y de proveerles de un entrenamiento en ambiente de altas aceleraciones, lo cual sólo se puede reproducir en vuelo real o en una centrífuga humana $(\mathrm{CH})^{8}$. Así queda recogido en la la Orden Ministerial N. ${ }^{\circ} 23 / 2011$ de 27 de abril (BOD N. ${ }^{\circ} 88$ de 6 de mayo de 2011) en el Capítulo VII, sobre Entrenamiento Aeromédico. Además, se contempla en el STANAG N. 3827 («Mínimos requerimientos para entrenamiento de las tripulaciones aéreas en ambiente de altas «G» sostenidas») y en el STANAG N. ${ }^{\circ} 3114$ («Aeromedical Training of Flight Personnel») .

El entrenamiento en $\mathrm{CH}$ lo vienen realizando todos los pilotos de reactores del Ejército del Aire (EA) desde 1985, inicialmente en la CH de la Real Fuerza Aérea Holandesa y desde septiembre del 2007 en la $\mathrm{CH}$ avanzada de la Fuerza Aérea Alemana.

Los textos clásicos de Medicina Aeroespacial describen las manifestaciones clínicas que sufre el hombre expuesto a altas fuerzas $\mathrm{Gz}$, que varían en función de la intensidad, duración y de la dirección en la que actúan sobre el hombre, y de su lectura se puede desprender que todos los pilotos presentan síntomas comunes ante un perfil de carga de aceleraciones semejante; sin embargo no se han encontrado estudios semejantes al que presentamos en el curso de un entrenamiento en $\mathrm{CH}$.

\section{OBJETIVOS}

En este trabajo se han establecido como objetivos: 1) Conocer la prevalencia de síntomas y signos clínicos en un colectivo de 81 pilotos militares, en el curso de su exposición a altas aceleraciones en una $\mathrm{CH}$ de nueva generación, y 2) Determinar la tolerancia natural individual a las fuerzas $\mathrm{Gz}$ en el curso del aumento gradual de las mismas.

\section{MATERIAL Y MÉTODOS}

Los datos que presentamos han sido obtenidos en el curso del entrenamiento realizado por pilotos alumnos de la Escuela de Reactores del EA en la Centrifuga Humana situada en la Base Aérea de Königsbruck en Baja Sajonia en Alemania.

\section{Características de la centrífuga}

La centrífuga humana consta de eje central vertical conectado al motor de conducción, sobre el que rota y del que parte un brazo rígido horizontal de 9,7 metros que rota sobre el eje y en cuyo extremo distal pivota una góndola. En el interior de la misma se reproduce la cabina del EF-2000, con un campo de visión de $180^{\circ}$ donde se coloca el piloto que dispone de la posibilidad de control activo de la $\mathrm{CH}$. Tiene una capacidad de generar aceleraciones longitudinales desde $-3 \mathrm{Gz}$ hasta $+12 \mathrm{Gz}$, aceleraciones transversas desde $-12 \mathrm{Gx}$ $\mathrm{a}+12 \mathrm{Gx}$ y aceleraciones laterales entre -6 Gy y $+4,9$ Gy.

\section{Sujetos}

Se sometieron a entrenamiento 81 pilotos alumnos de la escuela de reactores del EA, con una edad media de 22,7 $\pm 1,27$ años, un peso de 79,9 $\pm 8,4$ kilogramos y una talla de 180,3 $\pm 5,8$ centímetros, realizaron un total de 150 horas de vuelo, efectuando el entrenamiento básico en la centrífuga humana avanzada perteneciente a la Fuerza Aérea Alemana ${ }^{8}$ desde septiembre de 2007 hasta diciembre 2011.

\section{Características del entrenamiento}

Tiene una duración de dos días consecutivos, el primero de los cuales, los pilotos alumnos realizan ejercicios denominados de calentamiento y de práctica exponiéndose a niveles progresivamente crecientes de aceleraciones conseguidos con velocidad de comienzo (on-set rate) progresivamente más rápido, debiendo todos los pilotos conseguir tolerar un perfil de $+7 \mathrm{Gz}$ durante un mínimo de 15 segundos $^{9}$, conseguidos con un incremento de la velocidad de $6 \mathrm{G} /$ segundo, llevando el traje anti $\mathrm{G}$ conectado y realizando las maniobras de contracción muscular (AGSM: Anti-G Straining Maneuvers) especificas para aumentar la presión arterial cerebral en ambiente de altas fuerzas $\mathrm{Gz}^{10}$. Las características del programa de entrenamiento se detallan en la Tabla 1 .

Los entrenamientos están dirigidos por un oficial médico alemán y por un especialista en medicina aeroespacial del Centro de Instrucción de Medicina Aeroespacial (CIMA). Los datos recogidos en este estudio se obtienen de los registros obtenidos por el médico de vuelo mediante observación directa de las pruebas y a través de un cuestionario que se realiza a cada uno de los pilotos al final del segundo día de entrenamiento, lo cual ya ha sido descrito en una publicación anterior $^{8}$ La tolerancia individual a las fuerzas $\mathrm{Gz}$ se determina en cada piloto, exponiéndole a un aumento gradual de aceleraciones de $0,1 \mathrm{Gz}$ por segundo, teniendo el traje anti-G desactivado y sin que realice maniobras de contracción muscular, y se establece en el momento de aceleración en que cada piloto suelta la palanca de la cabina al considerar que está a punto de perder el conocimiento, generalmente coincidiendo con la aparición de visión negra.

\section{Estudio estadístico}

Estos datos, así como los síntomas y signos observados por el médico de vuelo o manifestados por el piloto, se incorporan como un conjunto de variables categóricas y cualitativas a una base de datos para su ulterior explotación analítica. En lo referente a la metodología estadística, como índices de tendencia central y de dispersión se emplearon para las variables cuantitativas, la media aritmética y la desviación estándar; y para las variables categóricas se emplearon las frecuencias absolutas y relativas expresadas en tanto por ciento. Para saber si la distribución de valores de la tolerancia relajada a $\mathrm{Gz}$ cumplía criterios de normalidad se aplicó el test de KolmogorovSmirnoff. Para la comparación de proporciones, se utilizó la prueba 
Tabla 1. Características del programa de entrenamiento básico de los alumnos de la Escuela de Reactores del Ejército del Aire en centrífuga humana.

Primer día

- Charla teórica sobre aceleraciones y sistemas/maniobras para incrementar la tolerancia de las mismas, familiarización con las características de la centrífuga y perfiles de entrenamiento

- Práctica de ejercicios de movilización, estiramiento y fortalecimiento de la columna cervical

- Monitorización electrocardiográfica (ECG)

- Práctica de las maniobras anti-G (AGSM: Anti-G Straining Maneuvers) y de la técnica respiratoria

Perfil de familiarización Giros partiendo de 1,4G (idle) hasta 3G, gradualmente primero y bruscamente después y vuelta a idle

PASIVO Comprobación de la tolerancia individual: Incrementos de 1G/10seg hasta la aparición de síntomas visuales. Sin pantalón anti-G ni maniobras AGSM

Entrenamiento básico Límites en 5G, y máximo $3 \mathrm{G} / \mathrm{seg}$; gradualmente, con pantalón antiG y maniobras AGSM. Mantener 5G 10 segundos.

ACTIVO* Una vez comprobada la técnica repetición con máximo on-set

Límites en $6 \mathrm{G} ; 3 \mathrm{G} / \mathrm{seg}$

Límites en 7G; 3 G/seg con máximo on-set, con pantalón antiG y maniobras AGSM. Mantener 7G 15 segundos: «perfil STANAG»

Segundo día

Entrenamiento AVANZADO Calentamiento: Límites en 5G con máximo 3 G/seg alcanzados primero gradualmente, con pantalón antiG y maniobras AGSM. Límites en $6 \mathrm{G} ; 3 \mathrm{G} / \mathrm{seg}$

Límites en $7 \mathrm{G} ; 3 \mathrm{G} / \mathrm{seg}$

Opcional: Límites en $8 \mathrm{G} ; 6 \mathrm{G} / \mathrm{seg}$

Entrenamiento

INTERACTIVO

Vuelo libre: Límites en $7 \mathrm{G} ; 6 \mathrm{G} / \mathrm{seg}$. Práctica de lo aprendido día anterior

«Dynamic Flight Simulator»: Mientras un alumno está en la centrífuga, con límites en 6 o $7 \mathrm{G}$ y 6 G/seg, otro maneja desde el centro de control un avión «blanco» realizando maniobras que deben ser seguidas por el primero

Conclusiones, recomendaciones y entrega de certificaciones

* De aquí en adelante todos son perfiles activos, lo que significa control de la aceleración por parte del piloto, previa fijación de los límites de G y de on-set rate (ritmo de aceleración en $\mathrm{G} / \mathrm{seg}$ ) por parte del instructor (de ahora en adelante todos los perfiles adaptados a la tolerancia individual).

de la Ji-Cuadrado. En todos los casos como grado de significación estadística, se empleó un valor de $\mathrm{p}<0,05$. La aplicación utilizada fue el paquete SPSS $^{\circledR}$ versión 15 para Windows (Chicago, Illinois).

\section{RESULTADOS}

Los síntomas y signos experimentados por los 81 pilotos en el curso de su exposición a $+7 \mathrm{Gz}$ durante 15 segundos, (on-set rate $6 \mathrm{G} /$ segundo) fueron los siguientes:

\section{Síntomas visuales}

Del total de los 81 pilotos entrenados, en el curso de la exposición a $+7 \mathrm{Gz}, 59(72,8 \%)$ de ellos refirieron haber presentado reducción concéntrica de los campos visuales o visión en túnel, $72(88,9$ $\%)$ visión borrosa o gris y $32(39,5 \%)$ reconocieron haber presentado ausencia total de visión o visión negra. El 65,4\% del total de la muestra reconocieron haber presentado visión gris y en túnel de forma simultánea (Figura 1).

\section{Dolores músculo-esqueléticos}

El aumento de las fuerzas $+\mathrm{Gz}$ supuso cambios hemodinámicos, desplazándose la sangre y fluidos corporales en sentido craneocaudal lo que se traduce en dolores musculares. En orden de frecuencia, 49 pilotos $(60,5 \%)$ refirieron dolor en antebrazos, 47 (58\%) dolor en muslos, 33 (40,7\%) dolor en brazos, 25 $(30,9 \%)$ dolor en cuello, $14(20,3 \%)$ dolor en tórax y el mismo porcentaje en los codos y $36(44,4 \%)$ presentaron dolor en otras zonas del cuerpo. La intensidad media del dolor fue valorada subjetivamente como moderada en la mayoría de los casos, siendo especialmente alta en brazos y codos (Figura 2).

\section{Hemorragias petequiales}

En el curso de la exposición repetida a 7 Gz, 90,1\% de los pilotos entrenados presentaron petequias y ocasionalmente hematomas subcutáneos, principalmente localizados en antebrazos, espalda y cara anterior del tórax por encima de la bolsa abdominal del traje anti-G (Figura 3).

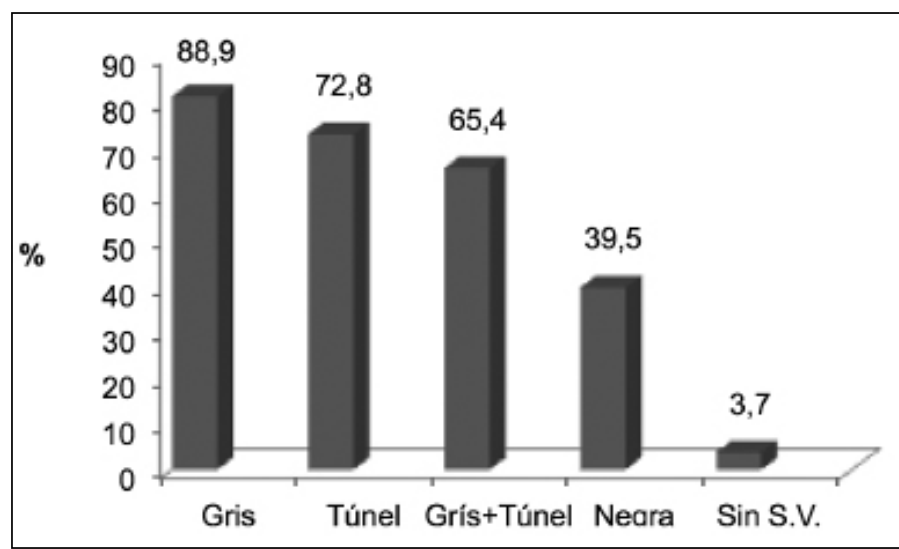

Figura 1. Porcentaje de pilotos con sintomas visuales (visión gris, visión en túnel y visión negra). 


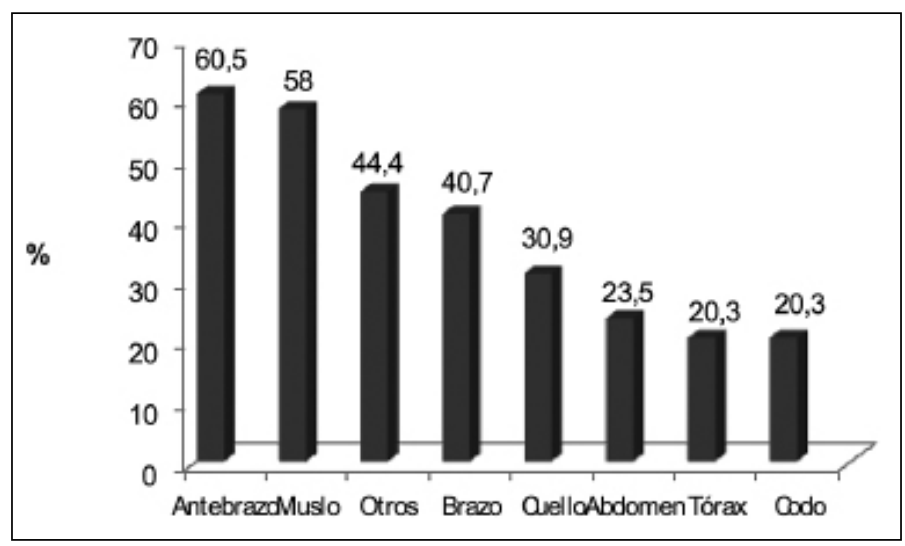

Figura 2. Porcentaje de pilotos con diversos dolores músculo-esqueléticos.

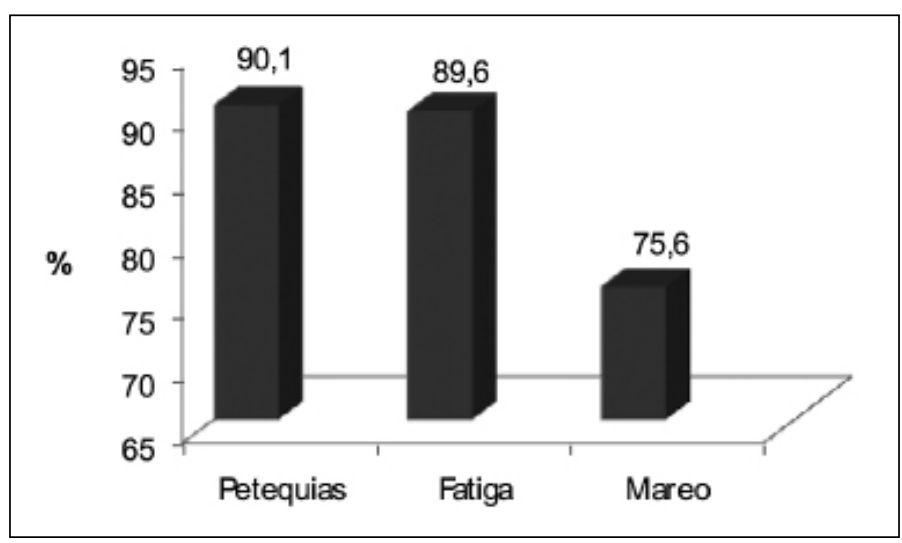

Figura 3. Porcentaje de pilotos con petequias, cansancio y desorientación espacial.

\section{Cansancio}

Setenta y dos pilotos $(89,6 \%)$ presentaron astenia intensa ya manifiesta tras el primer día de entrenamiento, que se acentuó tras el segundo día (Figura 3).

\section{Desorientación y vértigo}

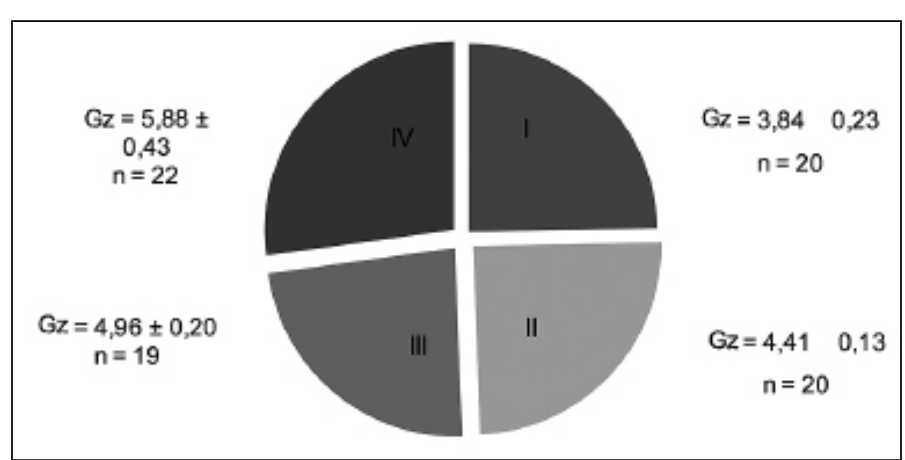

Figura 4. Distribución por cuartiles (media $\pm D S$ ) del grado de tolerancia a aceleraciones $+G z$. (Cuartil 1 de baja tolerancia; Cuartil 2 de tolerancia intermedia baja; Cuartil 3 de tolerancia media alta y Cuartil 4 de tolerancia alta).
La presentaron 61 de los 81 pilotos $(75,6 \%)$ como consecuencia del estímulo de los canales semicirculares. Esta sensación de inestabilidad en general de moderada intensidad $(4,33 \pm 2,02)$ persistió durante más de 15 minutos en el 53,3\%, remitiendo en el $46,7 \%$ restante en menos de los 15 minutos (Figura 3).

\section{Pérdida de conocimiento (G-LOC: G induced loss of consciousness)}

Dos de los 81 pilotos en el curso del entrenamiento sufrieron pérdida de conocimiento, uno a un valor de $5,94+\mathrm{Gz}$ y el otro a 6,5 $+\mathrm{Gz}$, situación que persistió durante 20 y 25 segundos respectivamente. De forma automática, al sufrir la pérdida de conocimiento (LOC), el piloto suelta la palanca y la centrífuga decelera hasta 1,4 Gz permitiendo el retorno de la sangre al cerebro, lo que desencadena convulsiones a nivel de la cabeza y extremidades. Además hubo otros 3 pilotos que sufrieron lo que se denomina «casi pérdida de conciencia» (aLOC: Almost loss of conciousness) durante periodos de tiempo de entre 10 y 15 segundos durante los cuales, aunque mantuvieron el tono muscular y la cabeza erguida, no percibieron las instrucciones que se les daba, actuaron descoordinadamente y presentaron después una amnesia de los hechos.

\section{Tolerancia relajada a fuerzas $+\mathbf{G z}$}

El grado de tolerancia a fuerzas $\mathrm{G}$ está en función de poder garantizar un adecuado suministro de sangre al cerebro. El método tradicional para determinar el nivel de tolerancia relajado a las fuerzas Gs en cada piloto se realiza con él sentado con el tronco erguido en la cabina de la góndola de la $\mathrm{CH}$, exponiéndole a un aumento gradual de las fuerzas $\mathrm{G}(0,1 \mathrm{G} /$ segundo), con el traje anti-G deshinchado y sin realizar las maniobras de contracción muscular. El nivel de tolerancia es variable en cada persona y se establece en el momento en que el piloto refiere pérdida de visión como consecuencia de la insuficiente perfusión ocular, sin menoscabo de que el flujo sanguíneo cerebral continúe manteniendo al individuo consciente y conservando la audición. En nuestra muestra de 81 pilotos, la tolerancia media relajada a las $\mathrm{Gs}$ fue de $4,79 \mathrm{Gz}( \pm 0,81)$ con un rango comprendido entre $3,40 \mathrm{G}$ y $6,86 \mathrm{G}$.

Con objeto de saber si el grado de tolerancia relajada a las $\mathrm{G}$ condicionaba la aparición de determinados síntomas, dividimos la muestra en cuatro cuartiles en función de su grado de tolerancia (Figura 4): Cuartil 1 de baja tolerancia (tolerancia relajada menor de $4,19 \mathrm{Gz}$ ), constituido por 20 pilotos; Cuartil 2 de tolerancia intermedia-baja (entre 4,19 y 4,68 Gz) constituido por 20 pilotos; Cuartil 3 de tolerancia media-alta (entre 4,58 y 5,40 Gz) constituido por 19 pilotos y Cuartil 4 de tolerancia alta (entre 5,40 y $6,86 \mathrm{Gz}$ ) formado por 20 pilotos.

El porcentaje de pilotos con visión negra en el primer y el cuarto cuartil, presenta frecuencias significativamente mayores $(p<0,05)$ que quienes lo presentan en los cuartiles segundo y tercero (Figura 5). No encontramos diferencias significativas entre el nivel de tolerancia relajada y la frecuencia de aparición de petequias, astenia, mareos ni dolores musculo-esqueléticos de ninguna localización. 


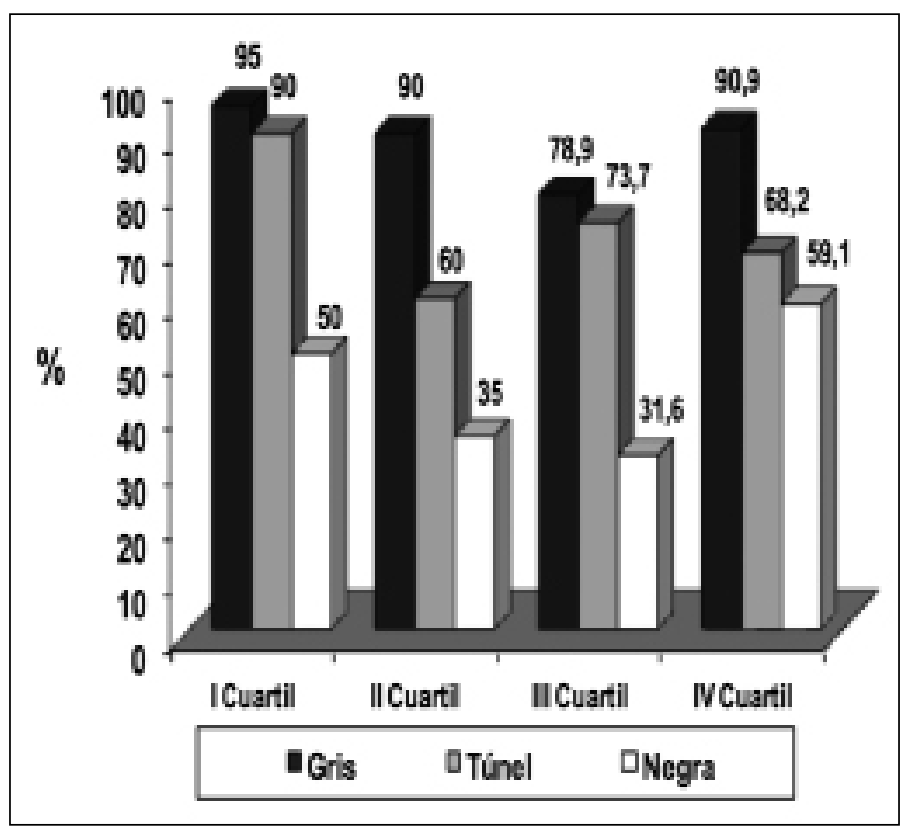

Figura 5. Distribución por cuartiles de las respuestas a las fuerzas + Gz sobre síntomas visuales: visión gris, en túnel y visión negra.

\section{DISCUSIÓN}

En el entrenamiento fisiológico se expone a los pilotos y tripulantes a situaciones ordinarias y extraordinarias de vuelo de forma controlada. Los más tradicionales como el entrenamiento a hipoxia de la altitud en cámara hipobárica o a desorientación espacial en vertífugo, no suele producir manifestaciones clínicas duraderas que persistan tras su finalización, ni dejar ninguna secuela tras el mis$\mathrm{mo}^{11}$, salvo cansancio o mareo ocasional que remite en pocas horas. La exposición a altas aceleraciones supone una situación mayor de estrés que desencadena una serie cambios en el organismo humano fisiológicamente adaptado a la gravedad terrestre, especialmente a nivel de sistema cardiovascular, de aparato respiratorio y sistema endocrino, con riesgo de pérdida de conocimiento ${ }^{12,13}$. En el presente estudio se han querido cuantificar los efectos del entrenamiento básico de 81 pilotos alumnos de la Escuela de Reactores del Ejército del Aire, que desde hace cuatro años realizan su entrenamiento en la centrífuga humana de la Fuerza Aérea Alemana que constituye un verdadero simulador dinámico de vuelo ${ }^{14}$. No se tiene conocimiento de otros trabajos en $\mathrm{CH}$ que hayan cuantificado la aparición de síntomas y signos en estos perfiles de aceleración.

En el curso de la exposición de los pilotos a $+7 \mathrm{Gz}$ durante 15 segundos, alcanzados con una velocidad de comienzo de $3 \mathrm{G} /$ segundo observamos que la casi totalidad de los pilotos experimentaron síntomas visuales, fundamentalmente visión gris producida por la disminución de la perfusión sanguínea a nivel de la retina como consecuencia de la disminución de la presión arterial a nivel de la arteria central de la retina y la resistencia ofrecida por la presión intraocular. Un menor número de pilotos refirió haber presentado visión en túnel producida por la pérdida de la visión periférica y en menor grado la visión central. Ambas situaciones fueron referidas de forma simultánea por el $65,4 \%$ de los pilotos. La visión negra consecuencia de la pérdida de visión periférica y central que suele ocurrir cuando la presión intraocular disminuye a aproximadamente $20 \mathrm{~mm}$ de $\mathrm{Hg}$, fue referida por $39,5 \%$ de los pilotos. Los síntomas visuales, son los primeros en manifestarse en el curso de la exposición a altas Gs, sin embargo 3 pilotos no reconocieron haberlos presentado, y la explicación que se da es que posiblemente los experimentaron de manera inadvertida.

Los dolores músculo-esqueléticos fueron referidos por muchos pilotos y son consecuencia de la acción de la propia gravedad y de los cambios hemodinámicos de sus lechos vasculares. También contribuyen las maniobras de contracción muscular en particular de las extremidades, del cuello, del cinturón escapular, del tórax y en particular de los antebrazos. El inflado a alta presión del traje anti-G se piensa que es responsable de que el $58 \%$ de los pilotos refiriera dolor en zona de muslos y el $23,5 \%$ en la zona abdominal.

Las pequeñas hemorragias cutáneas puntiformes aparecieron en las zonas de la piel que sufrieron mayor incremento de la presión arterial sin estar protegidas por los equipos anti-G. En nuestra muestra 73 de los 81 pilotos $(90,1 \%)$ presentaron petequias, principalmente en antebrazos, brazos, y en parte superior de abdomen y espalda por encima de la banda abdominal del traje anti-G. En la mayoría de los casos desaparecieron a las 8 horas, si bien en 6 casos se extendieron hasta formar amplios hematomas subcutáneos que persistieron más de 24 horas. Estas hemorragias son consecuencia de extravasación sanguínea o de la ruptura de los capilares por el gran aumento de la presión intravascular y que, en lenguaje aeronáutico, se conoce como «sarampión por las Gs»» ${ }^{1,15}$. La aparición de las mismas disminuye con la exposición reiterada a altas Gs y en centrífuga son siempre mayores.

El cansancio se asocia a las extenuantes maniobras de contracción muscular, principalmente isométricas que los pilotos tienen que realizar para mantener el flujo sanguíneo cerebral ${ }^{7}$ y fue referido por el $89,6 \%$ de los mismos ya que cada uno de ellos accedió a la $\mathrm{CH}$ al menos en cuatro ocasiones, y en cada una de ellas realizó distintas prácticas de entrenamiento a altas Gs.

La sensación de vértigo y desorientación referida por 75,6\% de los pilotos está relacionada con la variación de velocidad rotacional de la $\mathrm{CH}$ que conlleva cambios de ángulo de la góndola, generándose una aceleración tangencial con una fuerza tangencial inercial de igual intensidad que actúa en dirección opuesta. Para minimizar este vértigo, entre una prueba y otra no se llega a detener la $\mathrm{CH}$ que sigue girando rotada hacia el exterior sobre su eje longitudinal, reproduciendo un nivel de 1,4 Gz manteniendo la góndola un ángulo con respecto a la vertical. Esta sensación de vértigo persistió durante más de 15 minutos en más de la mitad de los pilotos.

La pérdida de conocimiento que sufrieron dos de los pilotos en el curso del entrenamiento fue debida a un insuficiente suministro de sangre al cerebro por debajo del nivel crítico necesario para mantener la conciencia, como consecuencia de una pobre realización de las maniobras de contracción muscular y/o de las técnicas de respiración, descartándose en ambos casos una baja tolerancia relajada individual a las aceleraciones. De forma automática, al sufrir la pérdida de conocimiento el piloto suelta la palanca y la centrífuga decelera hasta detenerse permitiendo el retorno de la sangre al cerebro lo que desencadena una serie de sacudidas convulsivas a nivel de la cabeza y extremidades superiores e inferiores, durante unos segundos durante los cuales el individuo permanece inconsciente, coincidiendo el final de las mismas con el regreso a la situación de conciencia.

En referencia a la tolerancia relajada a las fuerzas $\mathrm{G}$ se observa una amplia distribución de la población estudiada entre 3,40 G y un 
máximo de 6,86 G, con una media de 4,79 $( \pm 0,81) \mathrm{Gz}$. Dividiendo el total de la población en cuatro grupos semejantes en función de su tolerancia (baja, media-baja, media-alta, alta), con objeto de de ver si el mayor o menor grado de tolerancia condicionaba los síntomas antes descritos. Existen diferencias significativas $(\mathrm{p}<0,05)$ al comparar las proporciones entre los pilotos con tolerancia alta y baja $(59,1$ y $50 \%$ respectivamente) frente a los de tolerancia intermedia alta y baja (31,6 y $35 \%$, respectivamente). Solo se observa una mayor prevalencia de visión negra entre los pilotos con tolerancia alta y baja $(59,1$ y $50 \%$ respectivamente) frente a los de tolerancia intermedia alta y baja (31,6 y $35 \%$, respectivamente), lo que significa que los pilotos que han resistido mayor nivel de Gs han experimentado la pérdida de visión en mayor medida que aquéllos con una tolerancia intermedia. Por otro lado los pilotos con menor tolerancia son más susceptibles a la pérdida de visión en relación con una menor capacidad de mantener la perfusión cerebral, que aquellos con tolerancia intermedia. No se observa ninguna otra correlación entre el grado de tolerancia relajada a las Gs y la aparición de petequias, cansancio, desorientación o dolores musculares de distinta localización

\section{CONCLUSIONES}

- En el entrenamiento básico en centrífuga humana (nivel de $+7 \mathrm{Gz}$ durante 15 segundos) el 96,3\% de los pilotos, experimentó síntomas visuales, especialmente visión gris y túnel $\mathrm{y}$ en menor grado visión negra. Presentaron petequias en el $90,1 \%$ de los casos, astenia en el $88,6 \%$ y cinetosis en el $75,8 \%$.

- La tolerancia natural media relajada evaluada con un aumento gradual de $0,1 \mathrm{G}$ por segundo es de $4,79 \pm 0,81+\mathrm{Gz}$, con un rango entre 3,40 y $6,86+\mathrm{Gz}$.

- Los grupos de pilotos con mayor y menor tolerancia natural a las fuerzas $\mathrm{Gz}$, experimentaron visión negra con frecuencia significativamente mayor que aquéllos con tolerancia intermedia.

- No se encuentran diferencias significativas entre el nivel de tolerancia relajada a las fuerzas $\mathrm{G}$ y la frecuencia de aparición de petequias, astenia, mareos ni dolores musculo-esqueléticos. colaborado todos ellos en la administración de las encuestas que han permitido realizar el presente trabajo.

\section{BIBLIOGRAFÍA}

1. Green N.D.C. Effects of long-duration acceleration. En Ernsting's Aviation Medicine $4^{\mathrm{a}}$ edición. Oxford University Press, 2006; 137-158.

2. Alonso Rodríguez C. Evolución de la aviación de combate. Un desafío progresivo a la fisiología humana. Medicina Aeroespacial y Ambiental 2007: 106- 111.

3. Burton R.R:, Whinnery. Biodynamics: Sustained acceleration. En: Fundamentals of Aerospace Medicine $3^{\mathrm{a}}$ edición. Lippincott Williams \& Wilkins, 2002: 122153.

4. Alonso Rodríguez C, Ríos Tejada F. «Fisiología humana en ambiente de altas aceleraciones». Medicina Militar 1992; 48: 69-77.

5. Alonso Rodríguez C. «Ejercicios físicos para aumentar la tolerancia a las aceleraciones». Revista de Aeronaútica y Astronaútica 1986; 548: 879-885.

6. Navarro Ruiz V C, Alonso Rodríguez C. «Medicina Aeronaútica y Espacial». Tratado de Medicina Interna. Díaz-Rubio M, Espinós D. Sección XV. Madrid: Editorial Panamericana, 1994: Capítulo 298: 2041-2049.

7. Alonso Rodríguez C. «Medios para aumentar la tolerancia a las aceleraciones positivas». Revista de Aeronaútica y Astronaútica 1983: 516: 1025-1030.

8. Alonso Rodríguez C. Altas aceleraciones en aviación de Combate. Entrenamiento en centrífuga de última generación. Revista de Aeronáutica y Astronáutica 2008; 779: 1064- 1071 .

9. STANAG 3114 AMD (Edition 3). Aeromedical training of flight personnel NATO Standardization Agency. Brussels, 22 nov 2006.

10. Alonso Rodríguez C, Cantón Romero J J. «Medios actuales para evitar la pérdida de conciencia por altas aceleraciones». Revista de Aeronaútica y Astronaútica 1991; 609: 1180-1185.

11. Gradwell D.P. Prevention of hypoxia. En Ernsting's Aviation Medicine $4^{\mathrm{a}}$ edición. Oxford University Press 2006: 58-71.

12. Alonso Rodríguez C, Ríos Tejada F. «Pérdida de conocimiento en vuelo». Revista de Aeronaútica y Astronaútica 1989; 578: 240-243.

13. Alonso Rodríguez C. La Aviación Militar: Un reto para la Medicina Aeronáutica. Editorial de la Revista Medicina Aeroespacial y Ambiental 2005: 149-150.

14. Alonso Rodríguez C. «Eurofighter 2000, Soporte médico». Revista de Aeronaútica y Astronaútica 628; 1993: 1008-1013.

15. Hopkins P. Operating Typhoon. Presentado en el Six Nations Aeromedical Symposium. En RAF Centre of Aviation Medicine 27-28.02.2007.

\section{AGRADECIMIENTOS}

Al Coronel Director del Centro de Entrenamiento de Königsbrück, y en particular al personal encargado de la Centrífuga Humana por su profesionalidad y amabilidad, así como al personal del Servicio de Medicina Aeronáutica del CIMA y a todos los médicos de Vuelo de Unidades del Ejército del Aire que han acudido acompañando a los pilotos de reactores a estos entrenamientos habiendo 